\title{
VOIR LE MAL EN FACE : L'ORIGINE DU MONDE DE GUSTAVE COURBET
}

Frédérique Desbuissons

«Voilà ce que je voulais vous montrer, me dit Baudelaire, voilà le point de vue.»-Était-il assez bourgeois, hein! Qu'est-ce que c'est que des points de vue? Est-ce qu'il existe des points de vue ? ${ }^{1}$

L'œuvre de Gustave Courbet qu'il est aujourd'hui convenu de désigner sous le titre L'Origine $d u m_{0} e^{2}$ (fig.1) constitue un objet pictural atypique, difficile de faire coïncider avec les catégories habituelles de l'histoire de l'art; il échappe au champ traditionnel de la peinture, en résistant à ses classifications. Cette spécificité de l'œuvre de Courbet a marqué si durablement les manières de la considérer, que les effets s'en font sentir aujourd'hui encore.

Il convient, pour commencer, de revenir sur quelques signes révélant le statut d'exception de L'Origine du monde, avant de montrer, dans un second temps, comment ses commentateurs successifs sont parvenus, par une série de «négociations », à en rendre néanmoins compte comme d'une œuvre d'art : quelles stratégies particulières ont été élaborées afin de parler de cet objet inouï. Parmi ces commentateurs, le peintre lui-même contourné l'interdit de la représentation du sexe en peinture pour assurer, malgré tout, un statut d'œuvre d'art - et non de pornographie - à sa toile.

Cet article reprend l'essentiel de ma communication au colloque « Le Cul » organisé le 2 décembre 1995 au centre Saint-Charles de l'université de Paris I Panthéon-Sorbonne par Jean Da Silva et Michel Vanpeene. Il a été publié pour la première fois dans la revue Ligeia $n^{\circ} 19-20$, octobre 1996-juin 1997, p. 14-22, censuré de ses illustrations par le rédacteur en chef qui les jugaient «tristes, sales, gynécologiques », agrémenté d'images sans rapport avec son contenu ainsi que d'une foule de coquilles inédites et, last but not least, amputé de ses dernières notes de bas de page. Cette version rétablit mon texte dans sa forme et son illustration initiales. La bibliographie sur L'Origine du monde s'est considérablement accrue depuis 1996 et certains détails de mon argumentation mériteraient sans doute d'être revus et développés. Il n'en a toutefois pas perdu de sa pertinence. J'ai donc pris le parti de ne pas en réviser le contenu, me contentant d'en corriger seulement la ponctuation et quelques formulations maladroites.

Je tiens à remercier mesdames Isabelle Enault-Lechien, Hélène Toussaint et Michèle Haddad pour l'aide qu'elles m'ont à divers titres apportée au cours de cette recherche.

${ }^{1}$ Propos de Charles Baudelaire et commentaire de Gustave Courbet rapportés par Charles Léger dans Courbet, Paris, 1929, p.191.

${ }^{2}$ Gustave Courbet ne semble pas lui avoir attribué de titre. Jusque 1935, on le désigne par des périphrases ou comme «torse ». Lors d'une conférence prononcée le 14 décembre 1935au Kunsthaus de Zürich dont une copie du texte est conservée au centre de documentation du musée d'Orsay, Charles Léger emploie le titre «L'Origine du monde », semble-t-il pour la première fois. 
Chez son premier propriétaire (qui est aussi, peut-être, son commanditaire), la toile fait déjà figure de peinture d'exception. Khalil-Bey la place à part, puisqu'il ne l'accroche pas parmi la centaine d'œuvres qui composent sa galerie de tableaux mais dans son cabinet de toilette. C'est là que Gambetta la voit, à l'issue d'un dîner chez Khalil-Bey et en compagnie d'autres convives dont Gustave Courbet, de même que Maxime du Camp, qui en a décrit le mode si particulier d'exhibition, cachée derrière un rideau vert ${ }^{3}$.

Quand la collection Khalil-Bey est dispersée en vente publique en janvier 1868, le tableau en est soustrait pour des raisons qui ne peuvent qu'être de bienséance, tout comme il le sera de l'exposition posthume des œuvres de Courbet en 1882. Après avoir disparu plusieurs décennies, le tableau réapparaît en 1889. Ce n'est alors pas dans une galerie de peinture, mais chez un spécialiste d'un tout autre ordre, de La Narde : pas simplement antiquaire, comme on l'affirme trop souvent, mais marchand de chinoiseries et japonaiseries, établi 14 rue Saint-Georges ; c'est lui qui contacte Edmond de Goncourt pour, note ce dernier dans son Journal, lui montrer les curiosités qu'il vient de recevoir :

Aujourd'hui de La Narde m'a écrit qu'il avait reçu des livres et des objets japonais... J'y vais et pendant que je regarde, de deux yeux ennuyés, le médiocre envoi, de La Narde me dit: «Connaissez-vous cela? » Et il ouvre avec une clef un tableau, dont le panneau extérieur montre une église de village dans la neige et dont le panneau caché est le tableau peint par Courbet pour Khalil-Bey, un ventre de femme au noir et proéminent mont de Vénus, sur l'entrebâillement d'un con rose... ${ }^{4}$

... Venez donc voir mes estampes japonaises, lui aura dit le marchand. Il est difficile de situer avec précision la naissance de l'expression «montrer ses estampes japonaises » comme invitation explicite à l'acte sexuel, mais tout porte à croire que c'est précisément dans cette seconde moitié du $\mathrm{XIX}^{\mathrm{e}}$ qu'il conviendrait d'en dater l'apparition $^{5}$, quand la réouverture des échanges entre le Japon et l'Europe (1859) permet l'arrivée en masse, en Europe, d'estampes pour beaucoup érotiques ${ }^{6}$. Il faut voir plus qu'une coïncidence dans le fait que L'Origine $d u$ monde se trouve en vente précisément chez un marchand spécialisé dans ce type d'images : plus qu'un produit des traditionnels Beaux-Arts, c'est comme objet érotique, voire pornographique, que le tableau de Courbet est considéré et à ce titre soustrait du marché de l'art contemporain - vendu sous le manteau, comme une curiosité réservée à un amateur spécialisé. Ce qui explique, pour partie, que le tableau soit régulièrement qualifié de «petit », alors que ses dimensions, moyennes, sont de loin supérieures à celles des œuvres d'un Meissonier,

${ }^{3}$ Ludovic Halévy, Trois dîners avec Gambetta, publiés et annotés par Daniel Halévy, Paris, 1929, p.86-87. Maxime Du Camp, Les convulsions de Paris, Paris, 1878, t.2, p.263.

${ }^{4}$ Edmond et Jules de Goncourt, Journal. Mémoires de la vie littéraire, 29 juin 1889, Paris, 1989, vol.3, p.287.

${ }^{5}$ Claude Duneton en situe l'apparition au $\mathrm{XX}^{\mathrm{e}}$ siècle, ce qui semble tardif (C. Duneton en collab. avec Sylvie Claval, Le Bouquet des expressions imagées. Encyclopédie thématique des locutions figurées de la langue française, Paris, 1990, p.192-193).

${ }^{6}$ Timothy J. Clark, Ukiyo-e paintings in the British Museum, Londres, 1992, et sur les estampes érotiques plus particulièrement, Joseph-Marie Lo Duca, Histoire de l'érotisme, rééd. Paris, 1979, p.380-387. 
réputé pour ses formats réduits.

Sylviane de Decker Heftler a rapprochétrès judicieusement L'Origine du monde des daguerréotypes érotiques qui lui sont contemporains ou légèrement antérieurs, en montrant notamment que les uns comme l'autre sont plus des objets que des images plates : leur manipulation et parfois leur conservation en des sortes de reliquaires (des coffrets aux compartiments garnis de velours renfermant précieusement des daguerréotypes) les apparente à des fétiches, tandis que le dispositif d'exhibition du tableau de Courbet suppose lui aussi une manipulation, impliquant le corps entier, et non plus seulement l'œil désincarné, abstrait du reste du corps, qu'implique l'accrochage classique. Le geste du déplacement du rideau masquant la peinture réintroduit un corps entier, engagé dans une manipulation inséparable de l'acte de regarder (encore qu'une seconde fonction du rideau s'ajoute, nous le montrerons plus loin, contradictoirement à celle-ci). L'étroitesse du format, contribuant à la grande efficacité formelle de ces nus, est une autre grande proximité entre ces daguerréotypes et le tableau de Courbet ; le cadrage, rapproché, se concentre sur les particularités du corps, et plus spécialement sur certaines de ses parties isolées. Le daguerréotype, nous dit-elle, se prête ainsi mieux que la photographie à l'érotisation du corps ${ }^{7}$.

Aussi n'était-il pas question de faire paraître L'Origine du monde en public : depuis sa création et jusqu'en 1988, la toile demeure l'objet d'une jouissance privée, qui ne peuvent être exhibés publiquement - l'objet comme la jouissance. Seuls proches ou invités occasionnels auront l'occasion d'approcher l'œuvre de Courbet chez ses propriétaires successifs, au XIX ${ }^{\mathrm{e}}$ siècle comme au XX ${ }^{\mathrm{e}}-$ chez Khalil-Bey, puis à Budapest chez le baron Hatvany, et pour finir chez le couple Bataille-Lacan, propriétaires répertoriés auxquels il convient d'ajouter les marchands successifs dans les mains desquels le tableau est passé, ainsi que les militaires, nazis puis soviétiques, qui se le sont pour quelques temps approprié ${ }^{8}$. Les témoignages des rares personnes qui ont pu accéder à l'œuvre relèvent, eux aussi, d'un discours privé ou semi-privé : le Journal d'Edmond de Goncourt, les souvenirs de Maxime du Camp, etc.

Plus révélateur encore est le vocable utilisé par le critique et ami de Courbet, Jules Castagnary, dans ses notes préparatoires à une étude de l'œuvre de Courbet restée inachevée. D'abord dans la liste des nus de Courbet, puis dans celle des tableaux peints pour Khalil-Bey en 1866, L'Origine du monde est désigné comme «le vase de Khalil-Bey», «vase » étant un terme ordurier désignant le sexe féminin ${ }^{9}$. Comme si le langage courant ne convenait pas, et que seul l'argot pouvait nommer cet objetlà.

\footnotetext{
${ }^{7}$ Sylviane de DeckerHeftler, «Le nu photographique. Art impur, art réaliste », Photographies n ${ }^{\circ} 6$, décembre 1984, p.51.

${ }^{8}$ Linda Nochlin, «Courbet'sL'origine du monde : The Originwithout an Original », October $\mathrm{n}^{\circ} 37,1986, \mathrm{p} .79-81$.

${ }^{9}$ Nous remercions madame Hélène Toussaint de nous avoir signalé la présence de cette liste parmi les Papiers Courbet conservés au Département des Estampes et de la Photographie de la Bibliothèque nationale de France.
} 
Si cette toile a fait si longtemps exception, si on l'a, de fait, délibérément exclue du système des Beaux-Arts, c'est sans doute aussi, pour partie, parce que le genre d'inscription de ce tableau n'était pas sans ambiguïté.

Les historiens de l'art, quand ils s'attachent à une œuvre particulière, ont l'habitude de replacer celle-ci dans une série: série iconographique (à quels autres tableaux de même sujet peut-on la comparer ?), série générique (à quel genre pictural la rattacher ?) et, évidemment, série monographique (où la situer dans l'œuvre du peintre ?). Appliquée à L'Origine du monde, cette habitude s'avérera difficile à contenter ; elle révélera en outre la difficulté manifeste de le circonscrire.

C'est pourquoi un bon nombre d'historiens de l'art récents [article écrit en 1995] soit passent sous silence le tableau, soit ne font que le mentionner en contrepoint, mais ne lui accordent que rarement une place importante dans leur analyse ${ }^{10}$. On pourrait arguer qu'il est difficile de commenter une peinture invisible, si Les Casseurs de pierres, tout aussi invisible (mais pour cause de destruction $^{11}$ ), ne faisaient pas couramment partie de la plupart des études de l'œuvre du peintre. Pourtant, des nombreuses monographies publiées ces dix dernières années sur l'œuvre de Courbet, on remarquera l'absence, ou la grande discrétion, de L'Origine du monde.

Quant à la série iconographique, constatons qu'il est peu d'œuvres de l'histoire de l'art antérieur qui sauraient lui être comparées. Et notamment nul autre nu féminin fragmentaire articulé autour des organes génitaux dans l'histoire de l'art occidental avant 1866 - tout exemple postérieur étant alors, à raison, systématiquement rapportés au prototype de Courbet. Pour rencontrer avant 1866 une image comparable, il faut en fait sortir du strict cadre des Beaux-Arts ${ }^{12}$ et aller voir du côté de la pornographie, qui constitue d'ailleurs le domaine d'inscription constant du tableau de Courbet du XIX ${ }^{\mathrm{e}}$ au $\mathrm{XX}^{\mathrm{e}}$ siècle. La reproduction de L'Origine $d u$ monde sur sa couverture valut au roman de Jacques HenricAdorations perpétuelles les attaques des ligues de vertu de Besançon et de Clermont-Ferrand lors de sa parution en 1994, tandis qu'en 1995, un couple de touristes américains visitant le musée d'Orsay se détournait du tableau en déclarant qu'en Amérique, c'était de la pornographie ${ }^{13}$.

Les vues anatomiques offrent un second terrain de comparaison iconographique. Parmi celles, célèbres, de Jacques Gautier d'Agoty, on retiendra l'Hermaphrodite (1748) (fig.2), queson cadrage, sa disposition des membres et la place centrale accordée au sexe du modèle apparentent à L'Origine du

${ }^{10}$ À l'exception de Michèle Haddad, qui accorde au tableau une place centrale dans sa thèse inédite soutenue en 1994 sous la direction de Bruno Foucart à l'université Paris IV : «L'Art de Courbet. Transgressions/création. Sources».

${ }^{11}$ Les Casseurs de pierres, autrefois conservés au musée de Dresde, probablement détruits lors des bombardements de 1945. On n'en connaît plus qu'une version réduite conservée à la Fondation Reinhart à Winterthur.

${ }^{12}$ Alain Roger répertorie les quelques rares tableaux qui offrent des représentations de sexes féminins, sans qu'aucun d'eux ne se réduise au détail de Courbet: «Vulva, vultus, phallus », Communications n46, 1987, p.187. Repris dans L'art d'aimer ou la fascination de la féminité, Paris, 1995.

${ }^{13}$ À titre de comparaison, on se reportera aux photographies érotiques et pornographiques sélectionnées par Sylviane de DeckerHeftler (art. cité). La réaction des touristes est rapportée par Annette Vezin, «Sexe, Courbet et télévision », Info Matin, 5 septembre 1995. 
monde. De telles images demeurentau XIX ${ }^{\mathrm{e}}$ siècle des objets situés aux marges du système des BeauxArts - celui auquel le peintre se réfère durant toute sa carrière - et qui, en aucune façon, ne sont soumis aux mêmes jugements ni aux mêmes significations qu'une œuvre d'art traditionnelle.

Un autre exemple, lui aussi marginal, nous est offert par un ensemble de planches de l'architecte utopiste Jean-Jacques Lequeu à la fin du XVIII ${ }^{\mathrm{e}}$ siècle, qui forment une série consacrée au seul motif du sexe féminin cadré jusqu'à la naissance des cuisses ou plus rarement des seins(fig.3). Son projet se déploie aux limites des champs artistique et scientifique, la série constituant les débuts d'une sorte de classification typologique des vulves, à la manière d'une anatomie positiviste ${ }^{14}$. Là encore, il s'agit d'objets artistiques exceptionnellement atypiques.

Le narrateur d'un roman récent intitulé L'Origine du monde assimile le tableau de Courbet à une « peinture de harem ${ }^{15}$. Si aucun accessoire oriental ne permet de ranger ce nu dans la série, bien fournie, des scènes de gynécée qui se multiplient tout au long du XIX ${ }^{\mathrm{e}}$ siècle, le rapprochement n'est pas si incongru: comme Francis Haskell l'a montré, son commanditaire supposé, Khalil-Bey, possédait de telles peintures et sacrifiait volontiers au goût occidental pour les constructions idéologiques de l'Orient (femmes lascives et passives, luxe et voluptés efféminantes et autres mythes romantiques) ${ }^{16}$. Aussi a-t-on pu associer L'Origine du monde au Bain turc de Ingres qui figurait dans sa collection ${ }^{17}$. On ne trouvetoutefois pas, chez Courbet, le déplacement exotique fantasmatique, le pittoresque de pacotille - bref, l'éloignement - qui, dans la scène de harem, permet que l'on puisse en toute légitimité contempler des corps féminins désœuvrés, rassemblés là pour le seul plaisir des propriétaires fictif (le sultan) et réel (le collectionneur) qui coïncident dans le moment du regard. Faisant fi de l'hypocrisie de son siècle, L'Origine du monde évacue les accessoires autorisant le voyeurisme, qui n'était alors accepté qu'au prix d'un déplacement topologique : la scène se déroule ailleurs - autre temps (Antiquité), autre géographie (Orient), autre mode ou registre (Allégorie ou Mythologie). La plupart des récents commentateurs du tableau de Courbet ont insisté sur cette particularité : Françoise Gaillard estimant que «renonçant aux fards comme aux artifices de pose, il peint le sexe dans la vérité de sa nature », Jacqueline Lichtenstein parlant à son propos d'une «immédiateté de la présence de la chair en tant que chair», Laurence des Cars, pour laquelle «Courbet montre, sans recours à un prétexte mythologique ou littéraire, ce qu'aucun autre peintre moderne n'osera », Michèle Haddad, selon laquelle le peintre aurait bravé l'interdit sans avoir recours, comme Persée, à la protection du miroir ${ }^{18}$. L'erreur, à notre sens, de cette interprétation désormais

\footnotetext{
${ }^{14}$ Philippe Duboy, Jean-Jacques Lequeu, une énigme, Paris, 1987.

${ }^{15}$ Michel Boujut, L'origine du monde, [Paris], 1991, p.58.

${ }^{16}$ Francis Haskell, «Un Turc et ses tableaux dans le Paris du XIX ${ }^{\mathrm{e}}$ siècle » dans De l'art et du goût, jadis et naguère, Paris, 1987, p.362-383.

${ }^{17}$ Marcelin Pleynet, «Peinture et socialisme. Courbet public et privé », L'Infini n ${ }^{\circ}$, automne 1985, p.3-16. Repris dans Les modernes et la tradition, Paris, 1990.

${ }^{18}$ Françoise Gaillard, «Allégorie d'un fantasme fin de siècle, Courbet : L'Origine du monde », dans Philippe
} 
classique, est de considérer le tableau de Courbet comme le produit d'un « réalisme » sans concession. Nous montrerons au contraire que pour autant qu'elle élague le nu de ses accessoires, son œuvre n'est pas sans faire appel à un déplacement, mais d'un tout autre ordre.

La question du genre pictural de L'Origine $d u$ monde pourrait presque s'avérer moins problématique que celle de son sujet, si la conclusion à laquelle sont parvenus les commentateurs du tableau n'était si paradoxale: depuis le XIX ${ }^{\mathrm{e}}$ siècle, beaucoup s'accordent en effet à voir dans L'Origine du monde un portrait. En 1878, Du Camp parle à son propos d'un «portrait de femme bien difficile à décrire », expression que reprend à son compte en 1896Alexandre Estignard, pour lequel le « portrait de femme vue de face, difficile à décrire, que Courbet peignit pour un riche musulman [...] prouve que l'artiste ne savait pas se respecter et dégradait son métier jusqu'à l'abjection ». En 1991, le critique Philippe Muray rend compte en termes équivoque de sa rencontre estivale avec l'œuvre exposée au musée Gustave Courbet à Ornans : «Par le plus merveilleux des hasards, j'ai eu la joie, cet été, de me retrouver nez à nez avec ce que j'ai toujours considéré comme le chef-d'œuvre de Courbet ». Tout récemment encore, à l'occasion de son accrochage à l'exposition Féminin - Masculin, le sexe de l'art [1995-1996], un article anonyme paru dans Connaissance des Arts, ou encore Hervé Gauville dansLibération, développaient une semblable analyse : «le portrait de Courbet ampute son modèle d'à peu près tout le reste de son corps ». Robert Fernier avait pour sa part déjà mêlé astucieusement catégorisation générique et insertion monographique : examinant les nus féminins peints par Courbet dans les années 1860, dont L'Origine du monde représentaità ses yeux l'aboutissement, il concluait que « ces exercices de haute virtuosité préparaient admirablement Courbet au portrait ou, selon le cas, en étaient la résultante $»^{19}$.

Tout portrait, c'est une des caractéristiques du genre, suppose la personne particulière qui a posé et qu'un nom propre identifie : il s'agirait ici de Joanna Hiffernan (ou Heffernan), plus connue sous le diminutif de «Jo l'Irlandaise », du titre d'un tableau où Courbet l'a représentée coiffant sa chevelure rousse devant un miroir. C'est d'ailleurs ce portrait pudique que l'éditeur de Michel Boujut a choisi pour orner la couverture du roman éponyme racontant la quête tragique d'un amateur de Courbet dans le Paris de l'Occupation ${ }^{20}$. Jo Heffernan est dans les années 1850-1860 la maîtresse du peintre James Whistler, alors disciple de Courbet. On raconte que profitant du voyage de son jeune amant au Chili, l'Irlandaise aurait posé non seulement servi de modèle pour LesDormeuses, mais aussi

Hamon et Jean-Pierre Leduc-Adine (dir.), Mimesis et Semiosis. Littérature et représentation, Paris, 1992, p.429 ; Jacqueline Lichtenstein, "Histoire et variations d'un infini : le bleu entre le ciel et la terre », dans Azur, cat. exp. Jouy-en-Josas, Fondation Cartier, 1993, p.135 ; Laurence des Cars, 48/14, la revue du musée d'Orsay, $1^{\text {er }}$ septembre 1995, p.41; Michèle Haddad, «L'Art de Courbet. Transgressions/création. Sources », op. cit., p.208209.

${ }^{19}$ Maxime du Camp, op. cit., p.263 ; Alexandre Estignard, Courbet, sa vie, ses æeuvres, Besançon, 1896, p.57 ; Philippe Muray, «La peinture mise à nu », Art press n¹63, novembre 1991, p.40 ; «Courbet au grand jour », Connaissance des Arts, septembre 1995 ; Hervé Gauville, «Eros Center Georges Pompidou », Libération, 27 octobre 1995, p.28 ; Robert Fernier, Gustave Courbet. Peintre de l'Art Vivant, Paris, 1969, p.62.

${ }^{20}$ Michel Boujut, L'origine du monde, op. cit. 
pour L'Origine du monde. L'information pourrait bien être apocryphe et pour notre part nous n'en avons pas trouvé la source. Elle s'appuie sur le fait qu'en 1866, au moment supposé où Courbet peint L'Origine $d u$ monde et tandis que Whistler est à Valparaiso, Jo Hiffernan vient s'installer quelque temps chez Courbet à Paris. Les circonstances de ce séjour sont très mal connues; nos seules certitudessont que la jeune femme pose à cette occasion pour la femme blonde du Sommeil, et qu'à son retour, Whistler prend ses distances avec Courbet et le Réalisme. Reconnaître dans le nu de L'Origine $d u$ monde la rousse Irlandaise relève néanmoins, pour une bonne part, de l'autosuggestion.

La fonction de l'assimilation de L'Origine $d u$ monde au genre du portrait se comprend simplement, si l'on songe au travail de neutralisation mutuelle du visage et de la vulveanalysé par le philosophe Alain Roger, et que résume l'assertion «si le visage figure, il exclut la nature ${ }^{21}$, soit l'organe génital ${ }^{22}$. Considérer le tableau comme un portrait, c'est en ôter la charge subversive, le «spiritualiser». À l'encontre des historiens ayant affirmé que c'était la suppression du visage qui annihilait la dimension pornographique de L'Origine $d u$ monde, nous estimons au contraire que c'est son retour, en quelque sorte «subliminal », qui constitue l'une des procédures de «sauvegarde» du tableau par la métaphore. Son inscription dans le genre du portrait s'apparente, de ce point de vue, à l'allégorisation accomplie par son titre.

S'interroger sur le genre de l'œuvre amène à s'interroger sur son sexe et la construction de celui-ci. Si tous les commentaires sont sans ambiguïté - c'est bien d'une image féminine qu'il est question ici -, en revanche la question du sexe du spectateur (et nous rangerons le peintre dans cette catégorie) a été peu discutée.

On a remarqué à plusieurs reprises, en s'appuyant notamment sur les déclarations du peintre, que les compositions de Courbet s'articulaient souvent en séries ou en phases ${ }^{23}$. Castagnary reprend cette thématique dans ses notes sur l'œuvre du peintre, prévoyant de «Résumer les séries » et indiquant que «Courbet procède par séries/La forêt; chasses; animaux/La mer/Les fleurs/Les Parisiennes $»^{24}$. L'Origine du monde n'échapperait pas à cette logique, comme l'a estimé Alain Roger qui interprète L'Origine du monde comme le maillon d'une chaîne :Avant, Pendant, Après ${ }^{25}$. Cette interprétationest présente dès 1866. Le critique Charles Beauquier, qui tient la rubrique des potins artistiques dans la Revue littéraire de la Franche-Comté, rend alors compte de la «commande»

\footnotetext{
${ }^{21}$ Alain Roger, «Vulva, vultus, phallus », art. cité, p.183-187.

${ }^{22}$ Dictionnaire Littré, article « nature » $\mathrm{n}^{\circ} 23:$ « Les parties qui servent à la génération, surtout dans les femelles des animaux. »

${ }^{23}$ Lettre de Gustave Courbet à Francis Wey, 22 décembre 1854 (Bibliothèque nationale de France, Département des Estampes et de la Photographie, Papiers Courbet, boîte 8 ; lettre de Gustave Courbet à Champfleury, 8 mars 1855 (bibliothèque d'Art et d'Archéologie, Ms 168 [9]) ; lettre de Gustave Courbet à Alfred Bruyas, 14 mars 1855 (bibliothèque d'Art et d'Archéologie, Ms 216, II, pièce 98).

${ }^{24}$ Jules Castagnary, Notes manuscrites, dans Papiers Courbet, boîte 3.

${ }^{25}$ Alain Roger, Nus et paysages. Essai sur la fonction de l'art, Paris, 1978, p.255.
} 
venant d'être faite à Courbet par Khalil-Bey d'un pendant destiné à aller de pair avec $L e$ Sommeil(fig.4) :

\begin{abstract}
Le fameux tableau des Deux Femmes nues refusé à l'avant-dernière Exposition a été vendu par M. Courbet à un diplomate turc. Notre peintre fait en ce moment un pendant à ce tableau. Je n'ai pas besoin de vous dire qu'il est aussi peu décent que le premier.

Dame! pour un Turc ! 26 $^{26}$
\end{abstract}

Plus intéressante encore est l'anecdote célèbre rapportée par Jules Troubat: le tableau qui venait de faire scandale au Salon de 1864étant déjà vendu quand Khalil-Bey le remarque dans l'atelier du peintre, ce dernier lui aurait proposé de peindre «la suite » plutôt que la copie réclamée par le collectionneur. Et Troubat de conclure : «Il en résulta une série de tableaux et tableautins, qui se cachent dans quelque musée secret d'Europe ou d'Amérique ${ }^{27}$. Traditionnellement cette « suite » est comprise comme étant soit Le Sommeil, soit L'Origine du monde. Le terme de «tableautin» nous inciterait à pencher pour la seconde hypothèse.

Les trois compositions constitueraient ainsi un ensemble cohérent, une série à part entière dans l'œuvre de Courbet. Une série qu'on ne pourrait plus reconstituer aujourd'hui qu'a disparu le tableau du Salon de $1864^{28}$. Le Sommeil(appelé aussi Paresse et Luxure) de 1866appartient aujourd'hui aux collections du musée du Petit-Palais, à Paris, tandis que L'Origine du monde est entré récemment [1995] dans celles du musée d'Orsay ${ }^{29}$. La logique de cette série serait tant thématique que narrative : des épisodes se succédant en une chaîne temporelle, de Vénus et Psyché, où une femme dénudée vient en éveiller une seconde endormie, suivi de leur enlacement. La place de L'Origine du monde dans cette suite peut se comprendre diversement, comme précédant l'enlacement, ou le suivant. Mais quelle que soit sa place dans la série des trois compositions, sa spécificité apparaît évidente : c'est la seule des trois compositions où la figure est unique. Des deux femmes de Vénus et Psyché et du Sommeil, il n'en reste plus qu'une, réduite à un torse en vue rapprochée - en fait grandeur nature. Il serait tentant de comprendre cette disparition de la seconde femme comme un déplacement plutôt qu'une exclusion : posons l'hypothèse que le second protagoniste n'a pas disparu mais qu'il a seulement changé de place, et par là même de fonction. Quittant l'intérieur de la représentation, il est venu occuper la place du spectateur. La scène (fantasmatique) ainsi posée, le spectateur, peintre ou collectionneur - forcément masculin au $\mathrm{XIX}^{\mathrm{e}}$ siècle - s'identifie à ce second protagoniste féminin. Il s'agit là bien évidemment

\footnotetext{
${ }^{26}$ Charles Beauquier, «Chronique », Revue littéraire de la Franche-Comté vol.3, $1{ }^{\mathrm{er}}$ août 1866, p.468-472. Beauquier confond en fait Vénus et Psyché, refusé au Salon de 1864 et momentanément acheté par LepelCointet, et le Sommeil acquis par Khalil-Bey.

${ }^{27}$ Jules Troubat, Une amitié à la d'Arthez. Champfleury, Courbet - Max Buchon, suivi d'une conférence sur Sainte-Beuve, Paris, 1900, p.119-120.

${ }^{28}$ On en connaît toutefois une réplique peinte elle aussi en 1864 et mesurant 147 x $192 \mathrm{~cm}$, avec ajout d'un perroquet, et une répétition modifiée de 1866, de 77 x $100 \mathrm{~cm}$ conservée au Kunstmuseum de Berne.

${ }^{29}$ Robert Fernier, La vie et l'œuvre de Gustave Courbet. Catalogue raisonné, Lausanne/Paris, 1977-1978, n³70371 et 530-532-533.
} 
d'une construction imaginaire, et narrative, qu'autorise la série ${ }^{30}$.

Le scénario que nous venons de décrire n'est pas exceptionnel en littérature : les exemples sont légions dans des œuvres de fiction de personnages changeant de sexe [genre] afin d'approcher l'intimité de l'autre sexe, qui leur est ordinairement interdite. L'on songe bien évidemment au travestissement de l'héroïne de Théophile Gautier,Madeleine de Maupin, enThéodore afin de connaître « de l'intérieur » le comportement des hommes en l'absence de femmes (« ils » gardent les dehors de la civilité quand nous sommes là, mais que disent-ils, que font-ils derrière notre dos ?, se demande l'héroïne), mais aussi celui du jeune Masetto des Contes de Boccace, se faisant passer pour ce qu'il n'est pas, sourd et muet, pour pénétrer dans l'intimité d'un couvent de nonnes. Comme lorsque le sultan Aroun al-Rachid se déguise en mendiant afin d'observer ses sujets tels qu'ils se comportent quand l'étiquette ne les contient plus, il s'agit pour ces protagonistes d'avoir accès à une réalité étrangère que leur seule présence suffirait à faire disparaître, ou du moins à affecter. Le travestissement est ainsi un moyen d'accès au réel tel qu'il existerait indépendamment du sujet, qui pour cela doit se rendre «autre». Il ne vise nullement à le remettre en question, et encore moins à le transformer : le travestissement n'a ici rien de subversif, puisqu'en renversant un ordre il ne l'affecte pas mais le laisse en état ${ }^{31}$.

Ainsi en est-il de l'adoption par le peintre Courbet d'une identité féminine, lui donnant accès, de manière imaginaire, à une intimité «réservée » sans modifier les rapports et les rôles sexuels impartis à chacun des deux sexes dans la peinture érotique occidentale. Le spectateur mâle reste bien l'ultime destinataire du spectacle d'un corps féminin traité comme objet de plaisir - un corps sans tête, car comme le dit le proverbe, «Femme sans tête, tout en est bon $»^{32}$. Le travestissement aide à contourner l'interdit et protège de la menace qu'il supporte, tout au moins dans l'imaginaire ${ }^{33}$.

Si transgression il y a dans la toile de Courbet, elle ne concerne pas tant les identités sexuelles et les rôles impartis socialement aux uns et aux autres, que la représentation dès lors qu'il y a obscénité à figurer un corps féminin sexué en Occident. L'introduction d'une narration implicite dans le fonctionnement du tableau constitue ainsi une concession, mais aussi un moyen d'élargir les limites du représentable en peinture : de repousser les frontières du Réalisme. Le contexte narratif ainsi constitué vient autoriser etlégitimer un spectacle ordinairement soustrait à la vue masculine, qu'il s'agisse du visiteur du Salon ou du collectionneur qui réunit des tableaux pour son plaisir privé.

\footnotetext{
${ }^{30}$ Madeleine Kahn analyse quelques-uns de ces «travestissements narratifs » dans Narrative Transvestism: Rhetoric and Gender in the Eighteenth-Century English Novel, Ithaca, 1991.

${ }^{31}$ On trouvera une bonne synthèse des questions posées par l'analyse historique et sociale du travestissement dans Natalie Zemon Davis, Society and Culture in Early Modern France,trad. fr. par Marie-Noëlle Bourguet, Les Cultures du peuple. Rituels, savoirs et résistances, Paris, 1979, p.216-219.

${ }^{32}$ Dans Laure Beaumont-Maillet, La guerre des sexes, $X V^{e}-X I X^{e}$ siècles, Paris, 1984, p.9.

${ }^{33}$ Soit : menace de castration pour les hommes, et de défloration pour les femmes (Natalie Zemon David, op. cit., p.216).
} 
On peut à ce titre lui associer un second dispositif, le rideau de tissu vert derrière lequel la toile était dissimulée chez Khalil-Bey, destiné lui aussi à maintenir l'objet transgressif dans les limites de la représentation classique. Ce rideau peut être,bien sûr, considéré comme un accessoire traditionnel de dissimulation/dévoilement propre aux images érotiques ou pornographiques. Mais l'on peut aussi songer à une autre de ses fonctions, tout aussi séculaire : le rideau tiré constitue, depuis l'antiquité (l'on songe à Parrhasios), un topos figuratif destiné à marquer le tableau comme représentation. Depuis la Renaissance jusqu'au $\mathrm{XX}^{\mathrm{e}}$ siècle, ce motif intervient dans des peintures aussi diverses que la Vénus d'Urbino de Titien (1538, Florence), la Sainte-Famille de Rembrandt ( 1646, Cassel) ou les Femmes d'Alger de Delacroix (1833, Paris), pour signifier au regard qu'un seuil vient d'être franchi, au delà duquel il quitte le réel pour entrer dans l'espace de la représentation. Le motif du rideau tiré découvrant un spectacle peint redouble ainsi le cadre (classique) du tableau, dont la fonction est similaire ${ }^{34}$. Il affirme que le propos d'un sexe peint est autre que celui du sexe réel, que nous sommes ici dans la contemplation esthétique et non dans la pornographie - un terme qui, au XIX siècle, désigne des productions relevant de la reproduction matérielle sans médiation ${ }^{35}$.

Cette mise à distance, traditionnelle, de l'espace pictural par rapport au réel, vise à limiter ce qui a été, pour partie, l'ambition de Courbet : ne pas établir de distinction entre l'art et le réel. La seule anecdote que nous connaissions où sont rapportés les commentaires du peintre sur son œuvre se situe ainsi en totale contradiction avec les aspects que nous venons d'évoquer, et demeure conforme au projet réaliste d'abolition des frontières entre le réel et sa représentation. La scène se passe chez Khalil-Bey, où sont réunis ses invités, dont Gustave Courbet et Léon Gambetta, qui raconte :
Après le dîner, on était là, regardant... admirant... On s'épuisait en phrases enthousiastes : C'est merveilleux... [...]. Courbet ne bronchait pas... On recommençait... Cela durait depuis dix minutes. Courbet n'en avait pas assez. À la fin, on s'arrêta, on ne trouvait plus rien. Courbet alors de dire avec sa grosse voix grasseyante et traînante :
- Vous trouvez cela beau... et vous avez raison... Oui, cela est beau... Oui, cela est très
beau, et tenez, Titien, Véronèse, LEUR Raphaël, MOI-MÊME nous n'avons jamais rien fait de plus beau... ${ }^{36}$

Ainsi, ce qu'admireraient les invités ne serait pas le tableau peint mais le corps réel, un objet «naturel», présent sans écart dans la peinture. Dans cette perspective, non seulement aucune limite morale ne saurait être opposée à l'art du peintre - rien n'est a priori laid et tout peut être peint -, mais de plus nul objet n'échapperait aupouvoir du grand peintre- moi, Gustave Courbet, je suis capable de

\footnotetext{
${ }^{34}$ Jean-Claude Lebensztejn, « À partir du cadre », dans Le cadre et le socle dans l'art du XX $X^{e}$ siècle, cat. exp. Dijon, [1984] ; Louis Marin, «Le cadre de la représentation et quelques-unes de ses figures », Cahiers du musée national d'Art moderne $\mathrm{n}^{\circ 24}$, été 1988, p.62-81, repris dans De la représentation, Paris, 1993, p.342-63.

${ }^{35}$ On insistera aujourd'hui plutôt sur le caractère fictionnel des images pornographiques : Patrick Baudry, «Le spectacle de la pornographie », Ethnologie française vol.26, n², 1996, p.302-308.

${ }^{36}$ Propos rapportés par Ludovic Halévy, op. cit., p.86-87.
} 
tout peindre: il n'y aurait en quelque sorte pas de réserve à son immanence. Car l'artiste avait circonscrit clairement son champ d'action, en définissant la peinture comme « une langue toute physique, qui se compose, pour mots, de tous les objets visibles, un objet abstrait, non visible, non existant, n'est pas du domaine de la peinture $»^{37}$. Une tette définition pose ici problème, dans la mesure où l'une des caractéristiques du sexe féminin est précisément de ne pas ressortir du visible : soit que l'on considère qu'il n'y a, là, « rien à voir », soit que l'on estime que ses spécificités s'accordent peu avec la prédominance masculine accordée au spectaculaire. L'organe sexuel féminin constitue, par excellence, le point d'achoppement de la peinture réaliste - sa limite.

C'est pourquoi la question posée par L'Origine du monde semblait donc bien être, pour Maxime Du Camp, «jusqu'où peut aller le Réalisme ? » Ce tableau possède à ses yeux une fonction paradigmatique, résumant à lui seul la nature même du Réalisme et de son principal protagoniste, leur immoralité foncière. C'est en ce sens que l'homme de lettres dénonce L'Origine du monde comme étant «le dernier mot du réalisme ${ }^{38}$-son ultime aboutissement, ou, pour le dire autrement, sa fin : comme si L'Origine du monde avait été inscrite, en projet, dès les débuts de l'esthétique réaliste et qu'elle en constituait l'aboutissement logique, le point terminal.

Les contemporains du peintre avaient en effet, dès les années 1850, de considérer le Réalisme en terme d'obscénité. En 1863, lors du procès intenté à un certain Lauger, négociant d'images pornographiques en vues stéréoscopiques utilisant le procédé Dagron, l'accusateur public Genreau dénonçait la reproduction de corps féminins «dans des poses naturelles, soit debout, soit couchées, mais [...] nudités absolues, exposant au regard d'une manière provocatrice des objets qui doivent être effacés par l'art, quand ils ne sont pas voilés par la pudeur », et attribuait à « une école qui s'est intitulée réaliste » la responsabilité d'une telle obscénité. Il désignait alors implicitement Courbet en faisant allusion aux Demoiselles des bords de la Seine (1857), «ces nymphes d'une race jusqu'alors inconnue, dont les bords de la Seine ont gardé le triste souvenir ${ }^{39}$. Citons également, plus tardivement, l'emploi très particulier du vocable de « réalisme » par Émile Gros-Kost, commentant la rivalité auprès d'une jeune femme d'un prêtre et de son bedeau : "Elle se mit en tête de satisfaire à la fois ces deux coq à plumes noires - chacun d'eux bien entendu se regardant comme le seul favorisé [...]. Le bedeau entrant un jour dans la sacristie surprit le prêtre en train de causer réalisme avec la jeune femme $»^{40}$.

\footnotetext{
${ }^{37}$ Lettre de Gustave Courbet au Courrier du dimanche, 25 décembre 1861, rééd. sous le titre Peut-on enseigner l'art?, Caen, 1986.

${ }^{38}$ Maxime du Camp, op. cit., p.263-64. Dans la logique du Manet de Bataille, Michèle Haddad qualifie quant à elle L'Origine du monde de morceau manquant du nu féminin au XIX ${ }^{\mathrm{e}}$ siècle - aboutissement du lent processus de dévoilement dont elle dresse l'historique (La divine et l'impure. Le nu au XIX', Paris, 1990, p.25).

${ }^{39}$ L. Sassère, avocat, « Code des photographes », Moniteur de la photographie, 1er octobre 1863, p.111-112.

${ }^{40}$ [Émile] Gros-Kost, Courbet, souvenirs intimes, rééd. Neuchâtel, 1994 [1880], p.117.
} 
Aussi, dès 1855, soit plus d'une décennie avant L'Origine du monde, un critique commentait en ces termes L'Homme à la pipe qui figuraient à l'Exposition Universelle ${ }^{41}$ :

Rien de plus vrai, certes, que ce portrait, mais doit-on reproduire tout ce qui est vrai. Il n'y aurait pas de raison alors pour que... En vérité, M. Courbet, vous nous feriez dire des bêtises!

Deux ans auparavant, une caricature de Nadar (fig.5) laissait envisager, elle aussi, la pire éventualité après l'envoi des Baigneuses au Salon :

Mais maintenant que M. Courbet nous a fait voir sa lune, que diable pourra-t-il nous montrer l'année prochaine $?^{42}$

\section{ILLUSTRATIONS :}

1. Gustave Courbet, L'Origine du monde, $1866 . \mathrm{h} / \mathrm{t}, 46$ x $55 \mathrm{~cm}$. Paris, musée d'Orsay.

2. Jacques Gautier d'Agoty, planche supplémentaire à l'Anatomie de la tête en tableaux imprimés [...] de Duverney, Paris, 1748, figurant dans l'exemplaire conservé à la bibliothèque d'Histoire de la Médecine de l'université Paris Descartes

3. Jean-Jacques Lequeu, L'infâme Vénus couchée, posture lubrique d'après nature, pl. des Figures lascives et obscènes par Le Queu, Bibliothèque nationale de France, Département des Estampes et de la Photographie

4. Gustave Courbet, Le Sommeil, 1866. h/t, 135 x 200 cm. Paris, musée du Petit-Palais

5. Nadar, « Revue du deuxième trimestre de 1853 », Journal pour rire, 2 juillet 1853

\footnotetext{
${ }^{41}$ Ernest Gebaüer, Les Beaux-Arts à l'exposition de 1855, Paris, 1855, p.132-133.

${ }^{42}$ Nadar, «Revue du deuxième trimestre de 1853 », Journal pour rire, 2 juillet 1853. Repris dans Charles Léger, Courbet selon les caricatures et les images, Paris, 1920, p.21.
} 